\title{
Incidence and impact on outcomes of acute kidney injury after a stroke: a systematic review and meta-analysis
}

\author{
Julia Arnold ${ }^{1}$, Khai Ping $\mathrm{Ng}^{1}$, Don Sims ${ }^{2}$, Paramjit Gill ${ }^{3,4}$, Paul Cockwell ${ }^{1}$ and Charles Ferro ${ }^{1 *}$ (D)
}

\begin{abstract}
Background: Patients with chronic kidney disease have worse outcomes after stroke. However, the burden of acute kidney injury after stroke has not been extensively investigated.

Methods: We used MEDLINE and Embase to conduct a systematic review and meta-analysis of published studies that provided data on the risk of AKI and outcomes in adults after ischemic and hemorrhagic stroke. Pooled incidence was examined using the Stuart-Ord method in a DerSimonian-Laird model. Pooled Odds Ratios and 95\% confidence intervals were calculated for outcomes using a random effects model. This review was registered with PROSPERO (CRD42017064588).

Results: Eight studies were included, five from the United States, representing 99.9\% of included patients. Three studies used established acute kidney injury criteria based on creatinine values to define acute kidney injury and five used International Classification of Diseases coding definitions. Overall pooled incidence was 9.61\% (95\% confidence interval 8. 33-10.98). Incidence for studies using creatinine definitions was 19.51\% (95\% confidence interval 12.75-27.32\%) and for studies using coding definitions $4.63 \%$ (95\% confidence interval 3.65-5.72\%). Heterogeneity was high throughout. Mortality in stroke patients who sustained acute kidney injury was increased (Odds Ratio 2.45; 95\% confidence interval 1. 47-4.10). Three studies reported risk factors for acute kidney injury. There was sparse information on other outcomes.

Conclusions: Mortality in stroke patients who develop acute kidney injury is significantly increased. However the reported incidence of AKI after stroke varies widely and is underestimated using coding definitions. Larger international studies are required to identify potentially preventable factors to reduce acute kidney injury after stroke and improve outcomes.
\end{abstract}

Keywords: Acute kidney injury, Stroke, Cerebrovascular disease, Meta-analysis, Mortality/survival

\section{Background}

Stroke is the leading cause of neurological disability worldwide with huge social and economic impact [1]. In 2015 there were 6.24 million deaths caused by stroke [2]. Chronic kidney disease (CKD) is associated with an increased risk of stroke [3]. Some of this relates to shared traditional risk factors, for example hypertension, hypercholesterolemia, diabetes mellitus and cigarette smoking [4]. However CKD itself has also been recognized as a risk factor for stroke $[5,6]$. In a recent systematic review and meta-analysis comprising 83 studies and 30,392 strokes, Masson et al. demonstrated that

\footnotetext{
* Correspondence: Charles.Ferro@uhb.nhs.uk

'Department of Nephrology, University Hospitals Birmingham, Birmingham B15 2WB, UK

Full list of author information is available at the end of the article
}

stroke risk increased by $7 \%$ for every $10 \mathrm{mls} / \mathrm{min} / 1.73 \mathrm{~m}^{2}$ decline in glomerular filtration rate (GFR) [7].

Acute kidney injury (AKI) is a clinical syndrome defined as an abrupt decrease in kidney function resulting in disturbance of fluid, electrolyte and acid-base homeostasis [8]. AKI is a spectrum, ranging from mild, asymptomatic injury to severe injury requiring renal replacement therapy (RRT) $[8,9]$. Over the last decade, with the development and wide adoption of international classification systems [8-10], there has been an increasing amount of research into the incidence of AKI and its influence on adverse outcomes in both high and low income countries [11-14].

After a stroke, neurological deficit leading to dysphagia and physical disability, physiological effects including changes in blood pressure and cerebral salt wasting, as well

(C) The Author(s). 2018 Open Access This article is distributed under the terms of the Creative Commons Attribution 4.0 International License (http://creativecommons.org/licenses/by/4.0/), which permits unrestricted use, distribution, and 
as investigations and treatments, can all potentially contribute to the development of AKI. Furthermore, older, comorbid patients are at greatest risk of AKI [14]. Strategies to prevent AKI in stroke patients could therefore be of great importance. Although the association between CKD and stroke outcomes has been the subject of several systematic reviews and meta-analyses $[7,15]$, the relationship between AKI and stroke is much less clear. We therefore analyzed the reported rates of AKI incidence after a stroke and the associations between AKI and outcomes after a stroke.

\section{Methods}

Our systematic review was registered with PROSPERO (CRD42017064588) and we adhered to the PRISMA reporting statement [16]. A literature search of MEDLINE was performed from 1946 through to 30 June 2017 using relevant text words and medical subject headings acute kidney injury, acute kidney failure, acute renal failure, acute renal insufficiency, combined with stroke, cerebrovascular disorders and CVA or TIA. Embase was searched from 1974 to 30 June 2017, using the same medical subject headings for AKI as for MEDLINE, combined with cerebrovascular accident, cerebrovascular disease, cerebrovascular disorder, brain hemorrhage; brain infarction and stroke (for a detailed search strategy see Additional file 1). All searches were limited to human studies with no language restrictions. Authors manually reviewed the reference lists of retrieved articles for additional relevant studies.

\section{Study selection}

Study eligibility was determined using a standardized form (Additional file 2). Two authors, JA and KN, independently screened the list of studies generated by the search, with disagreements resolved by a third author, CF. Titles and abstracts of all studies were screened before obtaining full text versions of relevant studies. To improve generalizability, studies were included if they were a case control or cohort (prospective or retrospective) study and had a sample size greater than 500 adult subjects hospitalized with either an acute ischemic or hemorrhagic stroke [17]. Included studies had a clear statement regarding the definition of acute kidney injury - creatinine values alone were not sufficient. Subarachnoid hemorrhage was not included in this systematic review in view of the different aetiopathophysiology.

\section{Data collection and analysis}

Data was collected using a standardized proforma (Additional file 2) by JA and KN. The following study details were recorded: authors, year of publication, country of publication, type of study, clinical setting, sample size, patient characteristics (age, sex, ethnicity, and comorbidities), definition, type and severity of stroke and definition of AKI. Clinical parameters on admission, including serum creatinine and/or GFR, exposure to radiocontrast media, where specified and number of patients who developed AKI were recorded. Outcomes including mortality, disability, length of stay, re-stroke or cardiac events were also recorded.

Study quality assessment was performed independently by two authors, JA and CF using the Newcastle-Ottawa scale [18]. A maximum of 9 points can be allocated to a particular study based on quality of selection, comparability and study outcome (including follow up). Scores were defined as poor (0-3), fair (4-6) and good (7-9) [17].

Data synthesis, meta-analysis and statistical analysis were performed using Review Manager v5.3.5 software (The Cochrane Collaboration, UK) and StatsDirect v3.0 (StatsDirect Limited, UK). Meta-analysis of proportions was carried out using the Stuart-Ord (inverse double arcsine square root) method in a DerSimonian-Laird (random effects) model. The Odds Ratio (OR) with accompanying 95\% confidence intervals (95\% CI) were used to report individual and summary effect measures for dichotomous data. Chi squared tests for heterogeneity were performed to examine if the degrees of freedom were greater than the Cochran Q statistic, with $\alpha$ of below 0.05 considered to be statistically significant. In addition, the $\mathrm{I}^{2}$ statistic was calculated to provide the estimated percentage of heterogeneity observed. $\mathrm{I}^{2}$ values of $25 \%, 50 \%$ and $75 \%$ correspond to low, medium and high levels of heterogeneity. Any heterogeneity was further explored. A two-sided $P$ value of $<$ 0.05 was considered significant for all analyses.

\section{Results}

\section{Study characteristics}

A total of 6173 potentially relevant citations were identified (Fig. 1), of which 816 were duplicates. A further 5309 articles were excluded after review of title and abstract and an additional 40 excluded after full text review. The characteristics of the eight included studies are displayed in Table 1 [19-26] with the study outcomes summarized in Table 2. All eight studies were published in the English language between 2007 and 2015. Seven studies were considered to be of good quality and one of fair quality. The eight studies provided data on 12,325,652 patients (range 897 to 7,068,334) from four countries (five from the US [20, 22-24, 26], and one each from China [21], Greece [25] and Romania [19]). The US studies overwhelmingly had the largest sample sizes, with a total of 12,319,724 patients representing $99.9 \%$ of all included patients. Three of the US studies used Nationwide Inpatient Sample (NIS) data [23, 24, 26]. Although the same database was used, one study included only patients with ischemic stroke [23], one study included only patients with hemorrhagic stroke [24] and a third included only patients who sustained AKI requiring dialysis treatment (AKI-D) [26]. Therefore it was considered appropriate to include only the first two in the meta-analysis [23, 24]. Five out of eight studies used the International Classification of Diseases-9th or 10th Edition (ICD-9/1CD-10) coding to define AKI [2124] and AKI-D [26]. Only one of the studies reported data 


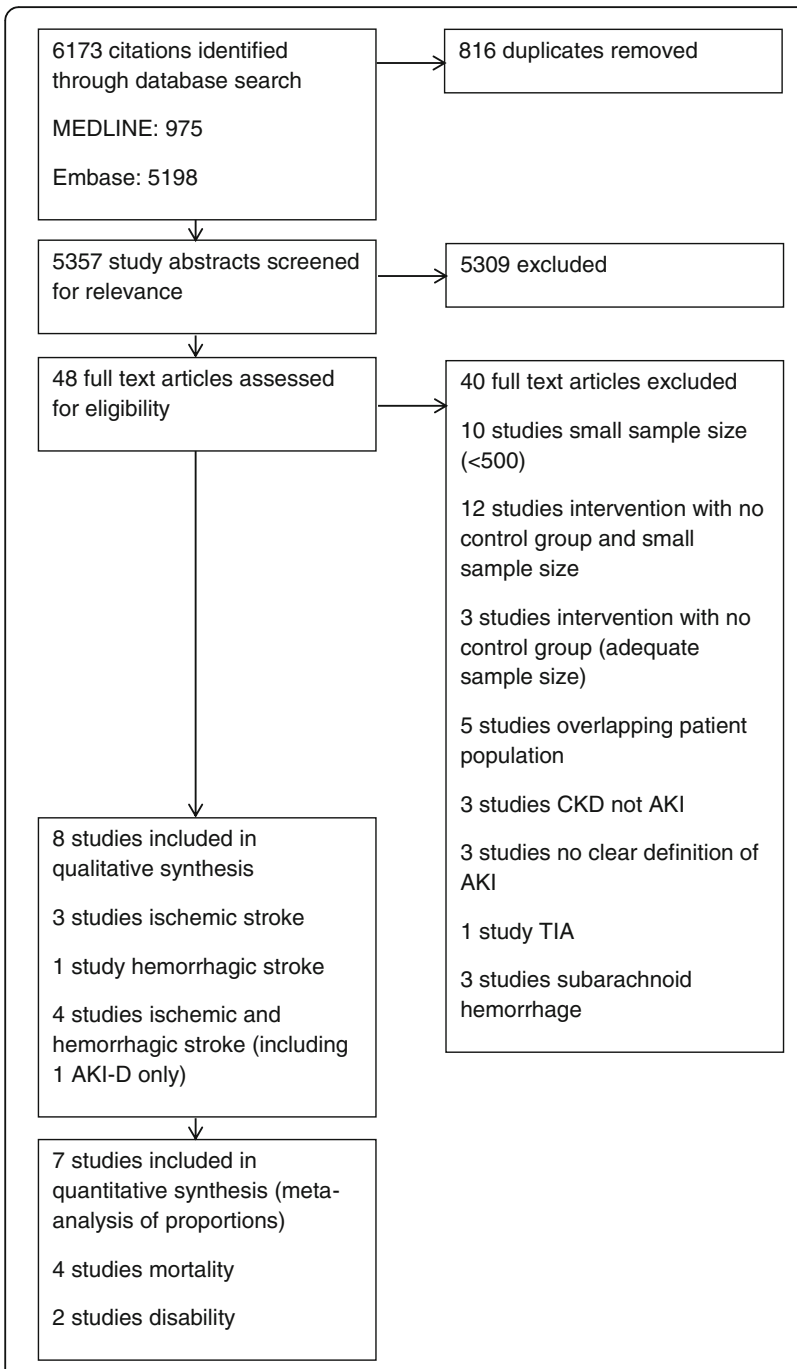

Fig. 1 PRISMA flow diagram for literature search and study selection

on AKI-D in addition to overall AKI incidence [24]. Two studies used the Acute Kidney Injury Network (AKIN) classification [20, 25] and one the Risk/ Injury/ Failure/ Loss/ End-stage (RIFLE) classification [19]. None used urine output criteria. Although there are some differences in the grading of AKI severity between these classifications, both define the absolute incidence of AKI as an increase in serum creatinine $>150 \%$. Stroke was determined using ICD coding in four studies [22-24, 26]. Three studies utilized the World Health Organization (WHO) definition of stroke [27] and extracted clinical data from medical records prospectively $[21,25]$ or retrospectively [19]. Two studies [21, 25] further subclassified the etiology of ischemic stroke using TOAST criteria [28]. One study utilized stroke registry data as well as clinical records and ICD coding [20].

Five studies followed patients until discharge from hospital [20, 22-24, 26], one for 30 days [19], one from hospitalization up to one year [21] and one from 30 days up to 10 years [25]
(Table 2). Two studies excluded patients with known CKD $[23,24]$ and two studies excluded patients with end-stage renal disease (ESRD) [20, 26]. Ischemic and hemorrhagic stroke patients were included in four of the studies [19, 20, 25, 26], ischemic stroke alone in three [21-23] and hemorrhagic stroke alone in one study [24] (Table 2).

\section{Pooled incidence of AKI after stroke}

Nadkarni et al. reported the incidence of AKI-D only [26]. This was $0.15 \%$ in hospitalizations with acute ischemic stroke and $0.35 \%$ in intracranial hemorrhage, with an overall incidence of $0.5 \%$. Saeed et al. reported an overall incidence of AKI-D of 1.7\% [24].

Using the remaining seven studies, the pooled proportion of AKI as a percentage was $9.61 \%$ (95\% CI 8.33-10.98) (Fig. 2) with an $\mathrm{I}^{2}$ statistic of $99.8 \%$ indicating high heterogeneity. Excluding Lin et al. [21], which reported a much lower incidence of AKI than any other study $(0.82 \%)$, made no difference to the heterogeneity ( $\left.\mathrm{I}^{2} 99.9 \%\right)$.

The pooled incidence of AKI in the studies that utilized ICD coding to define AKI [21-24] was 4.63\% (95\% CI 3.65$5.72 \%)$. Heterogeneity was high ( $\left.\mathrm{I}^{2} 99.9 \%\right)$. Excluding Lin et al. [21], the pooled incidence of AKI increased to 6.46\% (95\% CI $5.18-7.86 \%)$ and heterogeneity remained high $\left(\mathrm{I}^{2} 99.9 \%\right)$. Further excluding the study of hemorrhagic stroke hospitalizations [24], the pooled incidence of AKI remained similar (6.42\%; 95\% CI 4.07-9.27\%) with high heterogeneity ( $\mathrm{I}^{2}$ 90.6\%). In comparison, the pooled incidence in studies using creatinine-based AKI definitions [19, 20, 25] was 19.51\% (95\% CI 12.75-27.32\%), again with high heterogeneity ( $\left.\mathrm{I}^{2} 97.4 \%\right)$.

The pooled incidence of AKI in ischemic stroke [19-23, $25]$ was $9.62 \%$ (95\% CI $4.20-16.96 \%$; I I statistic $99.5 \%$ ). Excluding Lin et al. [21], AKI incidence increased to $12.45 \%$ (95\% CI 4.96-22.70\%) and heterogeneity remained high ( $\mathrm{I}^{2}$ 99.5\%). Using studies with a coding definition for AKI, the pooled incidence was 4.05\% (95\% CI 1.06-8.86\%; $\mathrm{I}^{2}$ statistic 99.1\%). Pooled incidence of AKI in studies utilizing creatinine-based definitions was $17.33 \%$ (95\% CI 9.4227.05\%) with high heterogeneity ( $\left.\mathrm{I}^{2} 97.6 \%\right)$.

The pooled incidence of AKI in hemorrhagic stroke $[19,20,24,25]$ was $19.17 \%$ (95\% CI 7.75-34.15\%). Heterogeneity was high ( $\left.\mathrm{I}^{2} 99.0 \%\right)$. Excluding Saeed et al. 2015 [24], the only study in this group to use a coding definition for AKI, the pooled incidence increased to $24.50 \%$ (95\% CI 18.03-31.61\%). Heterogeneity decreased but remained high $\left(\mathrm{I}^{2} 84.9 \%\right)$.

\section{Risk factors for AKI after stroke}

Factors associated with the development of AKI after multivariate analyses are shown in Table 2 . Three studies explored risk factors for the development of AKI after stroke $[19,20,25]$. Older age [19], worse renal function on admission [19, 20, 25], ischemic heart disease [19], heart failure 
Table 1 Characteristics of the 8 included studies

\begin{tabular}{|c|c|c|c|c|c|c|c|c|}
\hline Author & Type of study/ country & $\begin{array}{l}\text { No. of } \\
\text { subjects }\end{array}$ & Age (years) (SD) & $\begin{array}{l}\text { Men } \\
(\%)\end{array}$ & $\begin{array}{l}\text { Ischemic stroke cases } \\
(\%)\end{array}$ & $\begin{array}{l}\text { AKI } \\
\text { definition }\end{array}$ & $\begin{array}{l}\text { CKD } \\
\text { excluded? }\end{array}$ & $\begin{array}{l}\text { NOS } \\
\text { score }\end{array}$ \\
\hline $\begin{array}{l}\text { Covic et al., } \\
2008[19]\end{array}$ & $\begin{array}{l}\text { Observational, retrospective, } \\
\text { Romania }\end{array}$ & 1090 & $66.1 \pm 11.5$ & 49.3 & $932(85.5 \%)$ & $\begin{array}{l}\text { Creatinine } \\
\text { values; } \\
\text { RIFLE }\end{array}$ & No & $\frac{7}{(3,2,2)}$ \\
\hline $\begin{array}{l}\text { Khatri et al., } \\
2014[20]\end{array}$ & $\begin{array}{l}\text { Observational, retrospective, } \\
\text { United States }\end{array}$ & 1357 & $64 \pm 16$ & 56.0 & $528(38.9 \%)$ & $\begin{array}{l}\text { Creatinine } \\
\text { values; } \\
\text { AKIN }\end{array}$ & $\begin{array}{l}\text { GFR } \\
<15 \mathrm{ml} / \mathrm{min} \\
\text { excluded }\end{array}$ & $\begin{array}{l}6 \\
(2,2,2)\end{array}$ \\
\hline $\begin{array}{l}\text { Lin et al., } \\
2011[21]\end{array}$ & Observational, prospective, China & 2683 & $\begin{array}{l}66.1 \pm 13.59(\mathrm{AF}) \\
63.58 \pm 13.64 \\
\text { (no AF) }\end{array}$ & 58.4 & $2683(100 \%)$ & $\begin{array}{l}\text { ICD-10 } \\
\text { coding }\end{array}$ & No & $\begin{array}{l}7 \\
(3,2,2)\end{array}$ \\
\hline $\begin{array}{l}\text { Mohamed } \\
\text { et al.., } \\
2015 \text { [22] }\end{array}$ & $\begin{array}{l}\text { Observational, retrospective, } \\
\text { United States }\end{array}$ & 897 & $64.4 \pm 14.7$ & 44.0 & 897 (100\%) & $\begin{array}{l}\text { ICD-9 } \\
\text { coding }\end{array}$ & No & $\begin{array}{l}7 \\
(3,2,2)\end{array}$ \\
\hline $\begin{array}{l}\text { Saeed et al., } \\
2014 \text { [23] }\end{array}$ & $\begin{array}{l}\text { Observational, retrospective, United } \\
\text { States (Nationwide Inpatient } \\
\text { Sample data) }\end{array}$ & $7,068,334$ & $\begin{array}{l}\text { No AKI } 71 \pm 31 \\
\text { AKI } 74 \pm 28\end{array}$ & 46.1 & $7,068,334(100 \%)$ & $\begin{array}{l}\text { ICD-9 } \\
\text { coding }\end{array}$ & Yes & $\begin{array}{l}7 \\
(3,2,2)\end{array}$ \\
\hline $\begin{array}{l}\text { Saeed et al., } \\
2015 \text { [24] }\end{array}$ & $\begin{array}{l}\text { Observational, retrospective, United } \\
\text { States (Nationwide Inpatient } \\
\text { Sample data) }\end{array}$ & 614,454 & $\begin{array}{l}\text { No AKI } 69 \pm 37 \\
\text { AKI } 68 \pm 34\end{array}$ & 52.2 & $\begin{array}{l}0(0 \%) \text { (all cases were } \\
\text { hemorrhagic stroke) }\end{array}$ & $\begin{array}{l}\text { ICD-9 } \\
\text { coding }\end{array}$ & Yes & $\begin{array}{l}7 \\
(3,2,2)\end{array}$ \\
\hline $\begin{array}{l}\text { Tsagalis } \\
\text { et al., } \\
2008 \text { [25] }\end{array}$ & Observational, prospective, Greece & 2155 & $70.3 \pm 11.9$ & 61.2 & $1832(85 \%)$ & $\begin{array}{l}\text { Creatinine } \\
\text { values; } \\
\text { AKIN }\end{array}$ & No & $\begin{array}{l}8 \\
(4,2,2)\end{array}$ \\
\hline $\begin{array}{l}\text { Nadkarni } \\
\text { et al., } \\
2015 \text { [26] }\end{array}$ & $\begin{array}{l}\text { Observational, retrospective, United } \\
\text { States (Nationwide Inpatient } \\
\text { Sample data) }\end{array}$ & $4,634,682$ & $\begin{array}{l}\text { AIS } \\
\text { No AKI } 73 \pm 0.2 \\
\text { AKI } 66 \pm 0.3 \\
\text { ICH } \\
\text { No AKI } \\
69.7 \pm 0.13 \\
\text { AKI } 65.4 \pm 0.21\end{array}$ & $\begin{array}{l}\text { AIS } \\
50.0 \\
\mathrm{ICH} \\
60.0\end{array}$ & $3,937,928(85 \%)$ & $\begin{array}{l}\text { ICD-9 } \\
\text { coding } \\
\text { AKI-D only }\end{array}$ & No & $\begin{array}{l}7 \\
(3,2,2)\end{array}$ \\
\hline
\end{tabular}

Abbreviations: AIS, acute ischemic stroke; AKI, acute kidney injury; AKI-D, acute kidney injury requiring dialysis; AKIN, Acute Kidney Injury Network; CKD, chronic kidney disease; GFR, estimated glomerular filtration rate; ICD-9/ 10, International Classification of Diseases, 9th/ 10th Revision; ICH, intracranial hemorrhage; mls/ min, milliliters per minute; NOS, Newcastle-Ottawa Scale; RIFLE, Risk, Injury, Failure, Loss, End-Stage Renal Disease

$[19,25]$ and higher National Institutes of Health Stroke Scale (NIHSS) score on admission [20, 25] were all found to be associated the development of AKI in stroke patients. Use of angiotensin-converting enzyme inhibitors (ACEi) and angiotensin II receptor blockers (ARBs) were only marginally associated with AKI (OR 1.004; 95\% CI 0.993-1.058, $P=0.057$ ) in one study [19]. One study also tested the association between contrast-enhanced computerized tomography (CT) and AKI and found no relationship [20].

None of the studies presented data on the adjusted rates of AKI associated with cerebral angiography, thrombolysis or any vascular intervention (mechanical thrombectomy, carotid stenting or endarterectomy).

Two studies $[19,25]$ examined the relationship between stroke type and risk of developing AKI after adjustment for confounders. Covic et al. [19] reported an OR of 2.50 (95\% CI 1.42-4.41; $P=0.001$ ) in hemorrhagic stroke and Tsagalis et al. [25] an OR of 2.02 (95\% CI 1.34-3.04; P = 0.001 ) with lacunar stroke used as the reference.

\section{AKI and severity of stroke}

Two studies found an association between stroke severity (as determined by NIHSS score) and the development of AKI
[20, 25]. In Khatri et al. [20] the adjusted OR per 5 point increase in NIHSS score was 1.13 (95\% CI 1.07-1.19; $P<$ 0.001). Tsagalis et al. [25] reported an OR of 1.02 (95\% CI $1.01-1.03 ; P=0.020$ ) after adjustment for age, sex, presence of atrial fibrillation (AF), serum glucose, hematocrit and antihypertensive agent use in the first 48 hours of admission.

\section{AKI and degree of disability}

Five studies reported disability post stroke with varying definitions. Two studies [21, 22] recorded degree of disability post stroke, as measured by the modified Rankin Scale (mRS). However data from Lin et al. [21] could not be analyzed with respect to AKI. Mohamed et al. [22] found no association between AKI and degree of disability after multiple adjustments. Two studies [23, 24] used coded discharge destination from NIS data as a surrogate marker for disability. Discharge was categorized as none to minimal disability and any other discharge status (home health care, short-term hospital or other facility including intermediate care and skilled nursing home or death) as moderate to severe disability. Both studies found a higher incidence of moderate to severe disability in patients with AKI after adjustment for multiple confounders (Saeed et al. 2014, OR 1.3 (95\% CI 
Table 2 Incidence of AKl, associated factors, measured outcomes and adjustments in the 8 included studies

\begin{tabular}{|c|c|c|c|c|c|c|c|}
\hline Study & $\begin{array}{l}\text { Follow } \\
\text { up }\end{array}$ & $\begin{array}{l}\text { Factors associated } \\
\text { with AKI }\end{array}$ & $\begin{array}{l}\text { Crude } \\
\text { Mortality } \\
\text { in AKI }\end{array}$ & $\begin{array}{l}\text { AKI an independent risk factor } \\
\text { for mortality }\end{array}$ & Disability and AKI & $\begin{array}{l}\text { LOS (days) } \\
\text { and AKI }\end{array}$ & $\begin{array}{l}\text { Cost and } \\
\text { AKI }\end{array}$ \\
\hline $\begin{array}{l}\text { Covic et al., } \\
2008[19]\end{array}$ & 30 days & $\begin{array}{l}\text { Age, renal function } \\
\text { on admission, IHD, } \\
\text { CHF, hemorrhagic } \\
\text { stroke }\end{array}$ & $\begin{array}{l}43.1 \% \text { vs } \\
12.8 \% \\
(P=0.001)\end{array}$ & No & Not reported & Not reported & $\begin{array}{l}\text { Not } \\
\text { reported }\end{array}$ \\
\hline $\begin{array}{l}\text { Khatri et al., } \\
2014 \text { [20] }\end{array}$ & $\begin{array}{l}\text { Hospital } \\
\text { discharge }\end{array}$ & $\begin{array}{l}\text { Admission } \\
\text { creatinine, NIHSS } \\
\text { score }\end{array}$ & $\begin{array}{l}\text { AlS: } 33 \% \text { vs } \\
10 \% \\
(P \leq 0.001) \\
\text { ICH: } 40 \% \text { vs } \\
30 \% \\
(P=0.020)\end{array}$ & $\begin{array}{l}\text { For AIS only } \\
\text { OR } 3.08 \text { ( } 95 \% \text { CI 1.49-6.35, P= } 0.002 \text { ) } \\
\text { Adjusted for age, sex, race, } \\
\text { comorbidities, smoking, CTA, } \\
\text { creatinine, NIHSS score }\end{array}$ & Not reported & $\begin{array}{l}\text { Unadjusted } \\
\text { AlS: } 17.6 \text { vs } \\
8.4 \text { days } \\
(P \leq 0.001) \\
\mathrm{ICH}: 13.0 \text { vs } \\
8.0 \text { days } \\
(P \leq 0.001)\end{array}$ & $\begin{array}{l}\text { Not } \\
\text { reported }\end{array}$ \\
\hline $\begin{array}{l}\text { Lin et al., } \\
2011[21]\end{array}$ & 1 year & Not reported & $\begin{array}{l}\text { Not } \\
\text { reported }\end{array}$ & Not reported & Not reported & Not reported & $\begin{array}{l}\text { Not } \\
\text { reported }\end{array}$ \\
\hline $\begin{array}{l}\text { Mohamed } \\
\text { et al., } \\
2015 \text { [22] }\end{array}$ & $\begin{array}{l}\text { Hospital } \\
\text { discharge }\end{array}$ & Not reported & $\begin{array}{l}\text { Not } \\
\text { reported }\end{array}$ & Not reported & $\begin{array}{l}\text { Not significant after } \\
\text { adjustment }\end{array}$ & $\begin{array}{l}\text { OR } 2.63,95 \% \\
\text { Cl } 1.51-4.58 \\
\text { Adjusted for } \\
\text { comorbidities, } \\
\text { complications, } \\
\text { NIHSS score }\end{array}$ & $\begin{array}{l}\text { Not } \\
\text { reported }\end{array}$ \\
\hline $\begin{array}{l}\text { Saeed } \\
\text { et al., } \\
2014 \text { [23] }\end{array}$ & $\begin{array}{l}\text { Hospital } \\
\text { discharge }\end{array}$ & Not reported & $\begin{array}{l}8.4 \% \text { vs } 2.9 \% \\
(P \leq 0.001)\end{array}$ & $\begin{array}{l}\text { OR } 2.2 \text { ( } 95 \% \text { Cl 2.0-2.2, } P \leq 0.001 \text { ) } \\
\text { Adjusted for age, sex, race, } \\
\text { comorbidities, Gl bleeding, sepsis, } \\
\text { nicotine dependence }\end{array}$ & $\begin{array}{l}\text { OR for moderate/ } \\
\text { severe disability } \\
1.3(95 \% \mathrm{Cl} 1.3- \\
1.4, \mathrm{P} \leq 0.001) \\
\text { Adjusted as for } \\
\text { mortality }\end{array}$ & $\begin{array}{l}\text { Unadjusted } 6 \\
\text { vs } 4 \text { days } \\
(P<0.0001)\end{array}$ & $\begin{array}{l}\text { Unadjusted } \\
\text { USD } 38,613 \\
\text { vs } 24,474 \\
(P<0.0001)\end{array}$ \\
\hline $\begin{array}{l}\text { Saeed } \\
\text { et al., } \\
2015 \text { [24] }\end{array}$ & $\begin{array}{l}\text { Hospital } \\
\text { discharge }\end{array}$ & Not performed & $\begin{array}{l}\text { AKI: } 28.7 \% \text { vs } \\
22.4 \% \\
(P \leq 0.001) \\
\text { AKI-D vs AKI: } \\
50.2 \% \text { vs } \\
28.4 \% \\
(P \leq 0.001)\end{array}$ & $\begin{array}{l}\text { OR } 1.5 \text { ( } 95 \% \mathrm{Cl} 1.4-1.6, P \leq 0.001) \\
\text { Adjusted for age, sex, race, } \\
\text { comorbidities, nicotine dependence, } \\
\text { alcohol abuse, hospital bed size, } \\
\text { hospital teaching status }\end{array}$ & $\begin{array}{l}\text { OR for moderate/ } \\
\text { severe disability } \\
1.2(95 \% \mathrm{Cl} 1.1- \\
1.3, P \leq 0.001) \\
\text { Adjusted as for } \\
\text { mortality }\end{array}$ & $\begin{array}{l}\text { Unadjusted } \\
12 \text { vs } 7 \text { days } \\
(P<0.0001)\end{array}$ & $\begin{array}{l}\text { Unadjusted } \\
\text { USD } \\
104,142 \text { vs } \\
54,315 \\
(P<0.0001)\end{array}$ \\
\hline $\begin{array}{l}\text { Tsagalis } \\
\text { et al., } \\
2008 \text { [25] }\end{array}$ & 10 years & $\begin{array}{l}\text { NIHSS score, CHF, } \\
\text { ICH, GFR }\end{array}$ & $\begin{array}{l}\text { 30-day } \\
\text { mortality } \\
21.8 \% \text { vs } \\
12.5 \% \\
(P=0.001) \\
10-y e a r \\
\text { mortality } \\
75.9 \% \text { vs } \\
57.7 \% \\
(P=0.001)\end{array}$ & $\begin{array}{l}\text { 10-year HR } 1.24 \text { ( } 95 \% \text { Cl 1.07-1.44, } \\
\text { P } \leq 0.01 \text { ), } \\
\text { Adjusted for sex, SBP, hematocrit, } \\
\text { comorbidities, brain edema, } \\
\text { antihypertensives, statin use }\end{array}$ & Not reported & Not reported & $\begin{array}{l}\text { Not } \\
\text { reported }\end{array}$ \\
\hline $\begin{array}{l}\text { Nadkarni } \\
\text { et al., } \\
2015 \text { [26] } \\
\text { AKI-D only }\end{array}$ & $\begin{array}{l}\text { Hospital } \\
\text { discharge }\end{array}$ & Not performed & $\begin{array}{l}\text { AIS: } 31.8 \% \text { vs } \\
5.6 \% \\
(P \leq 0.01) \\
\text { ICH: } 40.4 \% \\
\text { vs } 28.5 \% \\
(P \leq 0.01)\end{array}$ & $\begin{array}{l}\text { AIS: OR } 1.30 \\
\text { (95\% CI 1.02-1.48, P } \leq 0.001) \\
\text { ICH: OR } 1.95 \\
\text { (95\% Cl 1.61-2.36, } P \leq 0.01) \\
\text { Adjusted for demographics, } \\
\text { hospital characteristics, Charlson } \\
\text { comorbidity index and other diagnoses }\end{array}$ & $\begin{array}{l}\text { OR for adverse } \\
\text { discharge } \\
\text { category } \\
\text { AIS: } 1.18,95 \% \mathrm{Cl} \\
1.02-1.37, P \leq 0.01 \\
\text { ICH: } 1.74 ; 95 \% \\
\mathrm{Cl} 1.34-2.24 \text {, } \\
P \leq 0.01 \\
\text { Adjusted as } \\
\text { for mortality }\end{array}$ & $\begin{array}{l}\text { Unadjusted } \\
\text { AlS: } 14.1 \text { vs } \\
3.6 \text { days } \\
(P \leq 0.01) \\
\text { ICH: } 23.5 \text { vs } \\
5.3 \text { days } \\
(P \leq 0.01)\end{array}$ & $\begin{array}{l}\text { Unadjusted } \\
\text { AIS: USD } \\
32,596 \text { vs } \\
8039 \\
(P \leq 0.01) \\
\text { ICH: USD } \\
58,111 \text { vs } \\
11,255 \\
(P \leq 0.01)\end{array}$ \\
\hline
\end{tabular}

Abbreviations: AF, atrial fibrillation; AIS, acute ischemic stroke; AKI, acute kidney injury; AKI-D, acute kidney injury requring dialysis; CHD, coronary heart disease; $\mathrm{CHF}$, congestive heart failure; $\mathrm{CT}$, computerized tomography; $\mathrm{CTA}$, computerized tomography angiography; GFR, glomerular filtration rate; Gl, gastrointestinal; ICH, intracranial hemorrhage; IHD, ischemic heart disease; LOS, length of stay; MI, myocardial infarction, mRS, modified Rankin Scale; NIHSS, National Institutes of Health Stroke Scale; OR, Odds Ratio; RIFLE, Risk, Injury, Failure, Loss, End-Stage Renal Disease; SBP, systolic blood pressure; TIA, transient ischemic attack; USD, United States Dollars

1.3-1.4, $P<0.0001$ ); Saeed et al. 2015, OR 1.2 (95\% CI 1.11.3, $\mathrm{P}<0.0001)$ ). A further study [26] utilized an 'adverse discharge' category to classify patients as being discharged to a nursing care facility, hospice or long-term care hospital. Here AKI-D was associated with increased odds of adverse discharge (adjusted OR 1.18, 95\% CI 1.02-1.37, $P<0.01$ for 


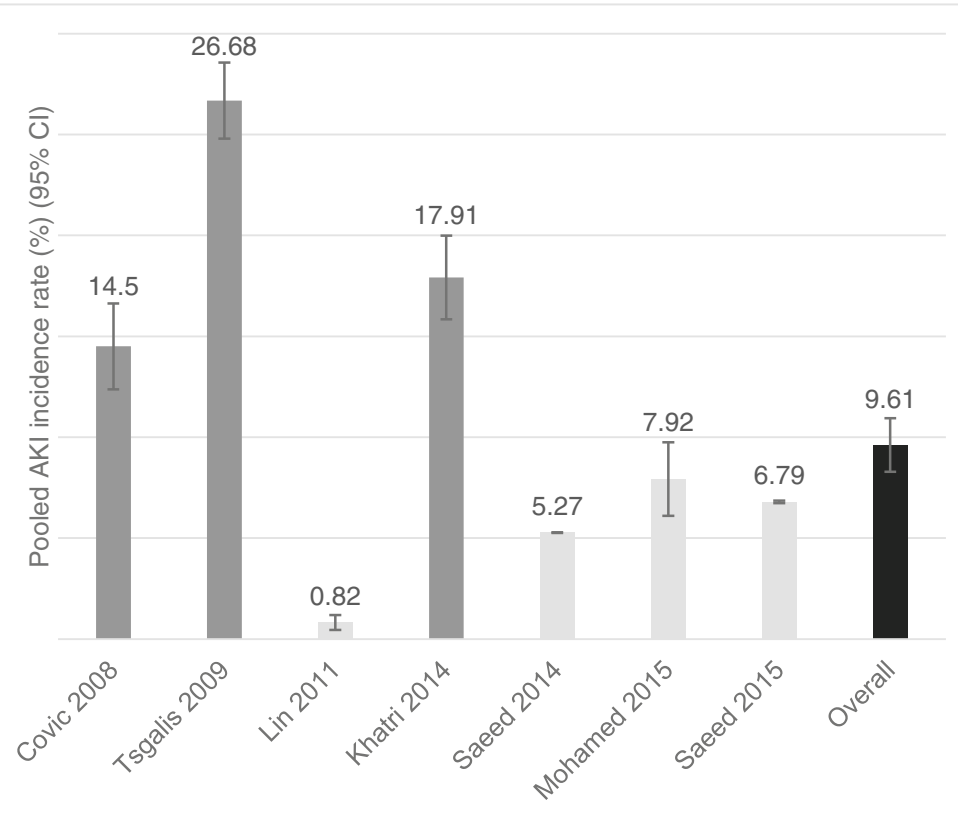

Studies using creatinine values

Studies using coding

Fig. 2 Pooled incidence rates of AKI in all studies listed by year. Data labels are percentages with 95\% Cl. AKI, acute kidney injury; 95\% $\mathrm{Cl}, 95 \%$ confidence interval

ischemic stroke, adjusted OR 1.74; 95\% CI, 1.34-2.24; $\mathrm{P}<$ 0.01 for $\mathrm{ICH}$ ) after adjusting for baseline demographics, hospital-level characteristics, Charlson comorbidity index and concurrent diagnoses.

\section{AKI and length of hospital stay and hospitalization costs} Five studies collected data on length of stay (LOS) [20, 22-24, 26]. All studies reported that AKI was associated with an increased length of stay ranging from 2 to 18 extra days spent in hospital (Table 2). In Mohamed et al. [22], this finding persisted after adjustment for age, NIHSS score, previous stroke and insurance status (no OR given, adjusted $P<0.0001$ ).

Three studies analyzed crude inpatient costs using NIS data $[23,24,26]$ and all showed AKI was associated with increased inpatients costs ranging from 14,139 to 49,827 US Dollars (Table 2).

\section{AKI and cardiovascular events}

One study [25] examined the relationship between AKI after a stroke and long-term cardiovascular events. The probability of having a composite cardiovascular event during the 10-year period was higher in the AKI group than the non-AKI group (cumulative probability 66.8 (95\% CI 56.6-76.9) vs 52.7 (95\% CI 48.5-56.1); $P=0.001$ ). In a Cox multivariable regression, AKI was an independent predictor of new composite cardiovascular events at 10 years (hazard ratio 1.22 ; 95\% CI 1.01-1.48, $P<0.05$ ) after adjustment for hypertension, diabetes, stroke subtypes, brain edema on imaging and hematocrit.

\section{AKI and post-thrombolytic ICH}

Saeed et al. 2014 [23] found that patients with AKI were more likely to suffer a post-thrombolytic ICH (OR 1.4; 95\% CI 1.3-1.6, $P<0.001)$ after multiple adjustments including age, sex, race/ ethnicity, hypertension, diabetes, AF, dyslipidemia, congestive heart failure, chronic lung disease, myocardial infarction, gastrointestinal bleeding, sepsis and nicotine dependence.

\section{AKI and mortality}

Six studies [19, 20, 23-26] compared mortality in AKI versus non-AKI groups with all reporting increased mortality in patients who developed AKI. Two studies reported higher crude mortality rates associated with severity of AKI $[19,20]$.

Four studies reported in-hospital mortality [20, 23, 24, $26]$ and two reported 30-day mortality [19, 25]. The OR for all-cause in-hospital mortality in patients with AKI was 2.11 (95\% CI 1.09-4.07) with high heterogeneity ( $\mathrm{I}^{2} 100 \%$; Fig. 3). Excluding Saeed et al. 2015, a study of ICH only, the OR increased to 2.67 (95\% CI 1.86-3.83) and heterogeneity decreased but remained high $\left(\mathrm{I}^{2} 84 \%\right)$. The OR for all-cause 30-day mortality in patients with AKI was 3.13 (95\% CI $1.20-8.19)$, again with high heterogeneity ( $\mathrm{I}^{2}$ 95\%; Fig. 3$)$. 


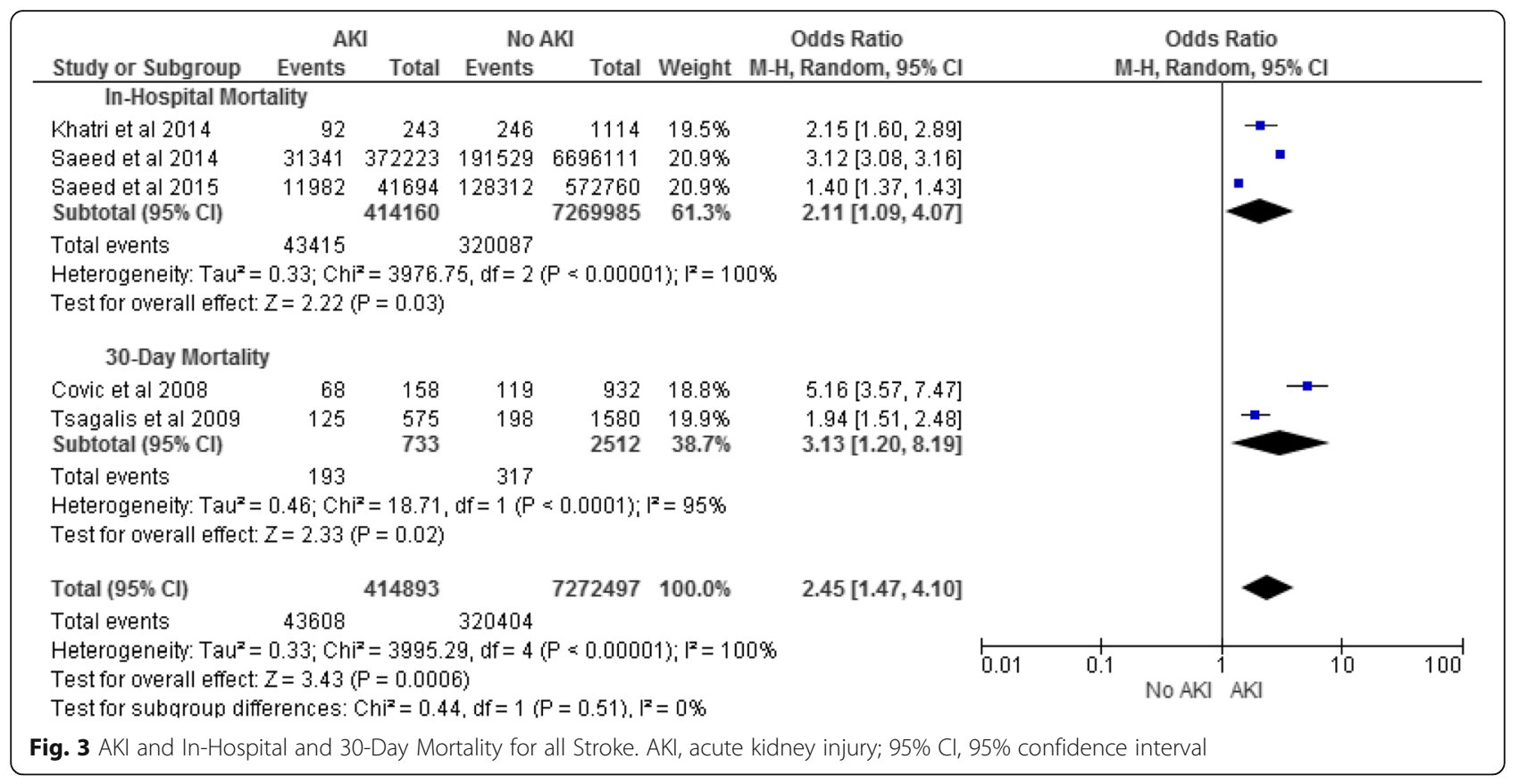

Two studies [20,23] provided data on in-hospital mortality after ischemic stroke with a pooled OR of 3.30 (95\% CI 2.56-4.26) and low heterogeneity (I ${ }^{2} 31 \%$; Fig. 4). Two studies $[20,24]$ provided data on in-hospital mortality after ICH with a pooled OR of 1.40 (95\% CI $1.37-$ 1.43 ) and low heterogeneity ( $\mathrm{I}^{2} 0 \%$; Fig. 4$)$.

Nadkarni et al. reported an adjusted OR for in-hospital mortality in AKI-D of 1.30 (95\% CI 1.12-1.48; $P<0.001)$ in ischemic stroke and 1.95 (95\% CI 1.61-2.36; $P<0.01)$ in ICH [26]. Since this study included AKI-D only it was excluded from the meta-analysis. Saeed et al. 2015 reported a higher crude mortality rate in patients with AKI-D than AKI without dialysis $(50.2 \%$ vs $28.4 \%, \mathrm{P}<0.001)$ [24].

Only one study provided long-term mortality data up to 10 years [25], demonstrating higher cumulative mortality in the AKI group at one year (34.6 vs 22.1 in non-AKI group) and 10 years (75.9 vs 57.7; $P=0.001$ ). In a Cox proportional hazards model AKI was an independent predictor of 10-year mortality (hazard ratio 1.24; 95\% CI $1.07-1.44, \mathrm{P}<0.01$ ) after adjustment for confounders including sex, hypertension, hypercholesterolemia, smoking, systolic blood pressure, brain edema, hematocrit, antihypertensive agent use after the event and ACEi/ARB and statin use on follow-up. The probability of 10-year mortality also increased with severity of AKI.

\section{Discussion}

We have shown that reported rates of AKI after stroke vary widely with a range of $0.82 \%$ to $26.68 \%$. Increased severity of stroke is related to the risk of AKI. Furthermore, having an episode of AKI after a stroke is associated with worse disability, increased inpatient mortality, LOS and cost, increased risk of future cardiovascular events and longer-term mortality.

There are marked differences in the reported incidence rates depending on the methodology used to identify patients with AKI. Using coding definitions, the pooled incidence of AKI was $4.63 \%$, compared with $19.51 \%$ for definitions based on serum creatinine values. ICD coding is known to underestimate the incidence of AKI $[29,30]$. Validation of ICD-9 codes for acute renal failure (ARF, which predates present use of the term AKI), used in Saeed et al. 2014 and 2015 are reported to have a sensitivity of $35.4 \%$ and a specificity of 97.7\%. Thus the low sensitivity for ARF codes may fail to identify patients with mild AKI that is more likely to go unrecognized and uncoded [30]. We know that severe AKI influences a number of outcomes, at high cost to individual patients, the health service and society [14, 31,32]. Given that milder categories of AKI are much more common they may be even more important to detect and prevent [11, 14, 33]. Furthermore, apart from Nadkarni et al. [26], only one other study reported data on the incidence of AKI-D and outcomes [24]. Since more severe AKI is related to worse outcomes, this is a significant limitation of our meta-analysis. Only three studies reported on the risk factors associated with AKI after stroke $[19,20,25]$ and generally confined themselves to reporting on those already known to be associated with AKI. Only two studies reported that stroke severity at presentation was associated with an increased risk of AKI [20, 25]. Two studies reported that hemorrhagic stroke type was associated with the development of AKI [20,25]. Interestingly, only one study [20] investigated the relationship between radiological contrast exposure and risk of AKI. None of the studies 


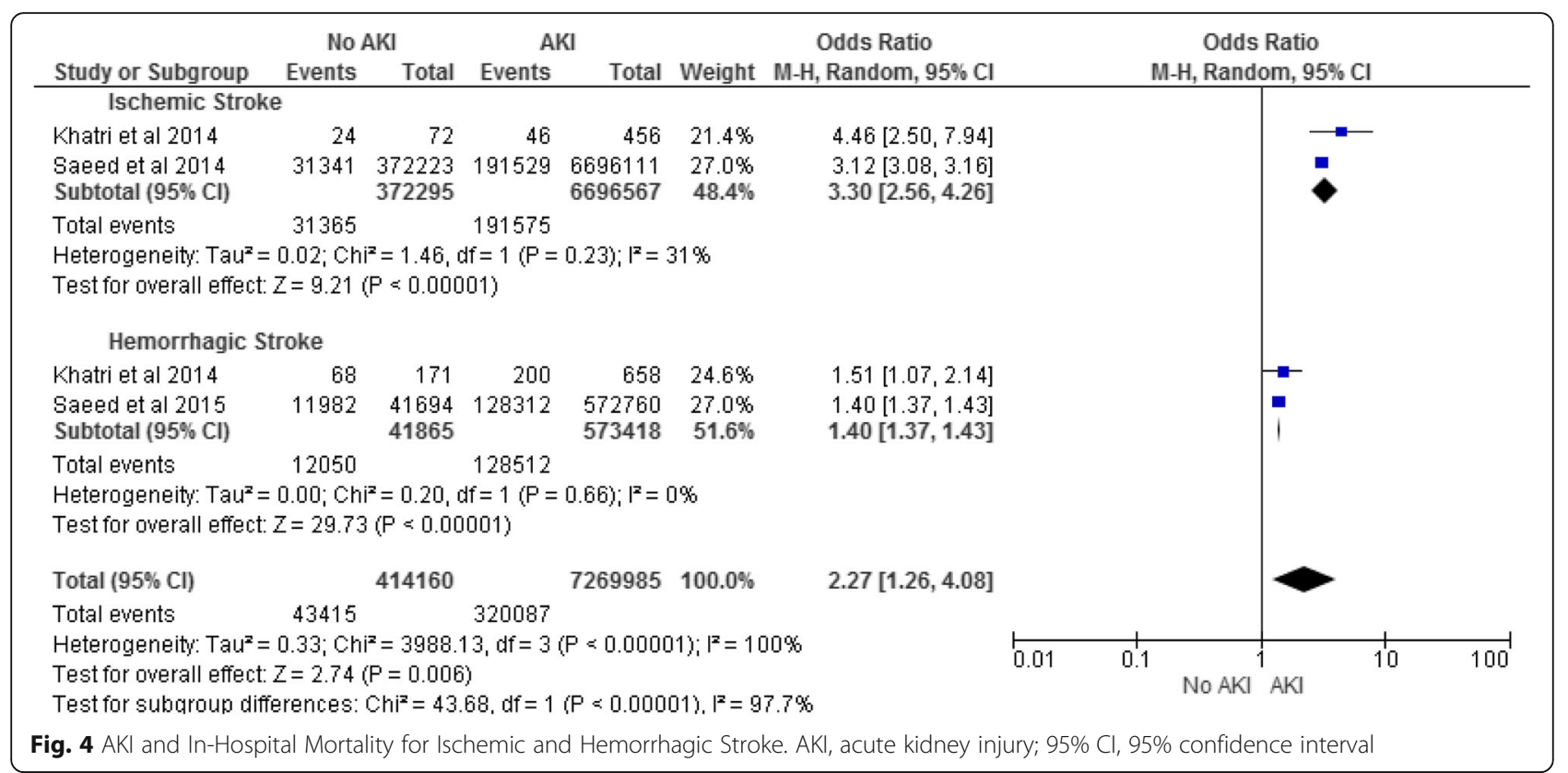

presented data on the association between thrombolysis, angiographic procedures or vascular intervention in ischemic stroke and AKI. In light of rapid advances in diagnostic scans and interventional treatments for stroke in recent years, including the use of intra-arterial thrombectomy [3], the incidence of AKI in stroke patients may well increase as these interventions become more widespread [3].

Lower baseline renal function was associated with an increased rate of AKI in three of the studies in this systematic review [19, 20, 25]. Patients with CKD are known to have poorer outcomes after a stroke [34-36]. This leads to the question of whether AKI adds further clinical relevance to what is already known about CKD and stroke. Of the studies that excluded patients with CKD [23, 24], AKI was still a clinically significant determinant of both disability and in-hospital mortality after adjustment for multiple confounding factors. Two further studies found AKI was still associated with worse outcomes after adjustment for baseline renal function or CKD category [20, 25]. This supports the theory that AKI is not merely an extension of the CKD spectrum and represents an important standalone factor that predicts worse outcomes in patients with acute stroke.

Our systematic review demonstrates a clear association between AKI and short-term mortality after stroke. This effect persisted after adjustment for CKD in three studies [19, 22, 26]. A further study [25] also found a relationship between AKI and long-term mortality, up to 10 years post stroke, after adjustment for CKD and post-stroke pharmacological treatments. This is consistent with the effect of AKI on short and long-term mortality in the context of other acute illnesses including myocardial infarction, sepsis and major surgery [37-41]. It is therefore, possible that interventions to prevent AKI may improve outcomes after stroke.
Our study has several strengths in that it encompassed several studies, all within the last decade with a large cumulative sample size and a large number of statistical adjustments. However, there are several significant limitations. Firstly, there was disproportionate representation of US data, accounting for $99.9 \%$ of the patient sample, which clearly affects generalizability of the estimates. There may also be significant ascertainment bias in view of the US being a high income country with increased availability of blood test monitoring and other diagnostic tests. Secondly, the number of studies included in the systematic review was small and those included in the meta-analysis smaller still. Despite sensitivity analyses, heterogeneity between studies remained substantial potentially suggesting that these studies should not be combined in a meta-analysis. However, we opted to present the data, warts and all, as this both highlights the need for further research in this area but also gives potential future researchers some idea of the numbers needed for recruitment into studies, as well as the rate and range of AKI to be expected using different definitions.

\section{Conclusions}

AKI appears to be a very common complication in hospitalized stroke patients and is associated with increased mortality, disability and healthcare costs. As a potentially preventable condition, further studies are needed in this area to attenuate the effects of AKI, including longer-term morbidity and mortality and the development of CKD. However in the first instance, additional representative studies, ideally using creatinine-based definitions of AKI are required to accurately determine point estimates of AKI post stroke and both short and long-term outcomes. 


\section{Additional files}

Additional file 1: Search Strategy. (DOCX $14 \mathrm{~kb}$ ).

Additional file 2: Data collection proforma. (DOCX 26 kb).

\section{Abbreviations}

ACEi: Angiotensin-converting enzyme inhibitor; AF: Atrial fibrillation; AKI: Acute kidney injury; AKI-D: Acute kidney injury requiring dialysis treatment; AKIN: Acute Kidney Injury Network; ARB: Angiotensin II receptor blocker; ARF: Acute renal failure; CKD: Chronic kidney disease; CT: Computerized tomography; CVA: Cerebrovascular accident; ESRD: End-stage renal disease; GFR: Glomerular filtration rate; ICD-9/10: International Classification of Diseases9th/10th Edition; ICH: Intracranial hemorrhage; IHD: Ischemic heart disease; mRS: modified Rankin Scale; NIHSS: National Institutes of Health Stroke Scale; NIS: Nationwide Inpatient Sample; OR: Odds ratio; PRISMA: Preferred Reporting Items for Systematic Reviews and Meta-Analyses; PROSPERO: International prospective register of systematic reviews; RIFLE: Risk/ Injury/ Failure/ Loss/ End-stage; RRT: Renal replacement therapy; TIA: Transient ischemic attack; TOAST: Trial of ORG 10172 in Acute Stroke Treatment; WHO: World Health Organization

\section{Acknowledgements}

We would like to acknowledge and thank Peter Nightingale of University Hospitals Birmingham NHS Foundation Trust for his statistical expertise and input in analysis for this systematic review.

\section{Availability of data and materials}

All data generated and/or analyzed during this systematic review are included in the published article and its supplementary information files.

\section{Authors' contributions}

JA and KN devised the search strategy and collected data, overseen by CF. JA and CF carried out quality assessment of the studies, synthesized data and conducted the meta-analysis. KN, DS, PG and PC interpreted the results and contributed to the manuscript. All authors read and approved the final version

\section{Ethics approval and consent to participate}

Not applicable.

\section{Consent for publication}

Not applicable.

\section{Competing interests}

The authors declare that they have no competing interests.

\section{Publisher's Note}

Springer Nature remains neutral with regard to jurisdictional claims in published maps and institutional affiliations.

\section{Author details}

'Department of Nephrology, University Hospitals Birmingham, Birmingham B15 2WB, UK. ²Department of Stroke, University Hospitals Birmingham, Birmingham, UK. ${ }^{3}$ Institute of Applied Health Research, College of Medical and Dental Sciences, University of Birmingham, Birmingham, UK. ${ }^{4}$ Warwick Medical School, University of Warwick, Coventry, UK.

Received: 19 December 2017 Accepted: 8 October 2018 Published online: 22 October 2018

\section{References}

1. Prabhakaran S, Ruff I, Bernstein RA. Acute stroke intervention: a systematic review. JAMA. 2015;313(14):1451-62.

2. Organisation, W.H. WHO Mortality Database. [cited 9 March 2017; Available from: http://www.who.int/healthinfo/mortality_data/en/.

3. Arnold J, Sims D. Ferro CJ. Modulation of stroke risk in chronic kidney disease. Clin Kidney J. 2016;9(1):29-38.
4. Writing Group Members, et al. Heart disease and stroke Statistics-2016 update: a report from the American Heart Association. Circulation. 2016; 133(4):e38-360.

5. Tomiyama H, Yamashina A. Clinical considerations for the association between vascular damage and chronic kidney disease. Pulse (Basel). 2014; 2(1-4):81-94.

6. Murray AM. The brain and the kidney connection: a model of accelerated vascular cognitive impairment. Neurology. 2009;73(12):916-7.

7. Masson P, et al. Chronic kidney disease and the risk of stroke: a systematic review and meta-analysis. Nephrol Dial Transplant. 2015;30(7):1162-9.

8. KDIGO. Clinical practice guideline for acute kidney injury. Kidney Int Suppl. 2012;2(1):1-138.

9. Mehta RL, et al. Acute kidney injury network: report of an initiative to improve outcomes in acute kidney injury. Crit Care. 2007;11(2):R31.

10. Bellomo R, et al. Acute renal failure - definition, outcome measures, animal models, fluid therapy and information technology needs: the second international consensus conference of the acute Dialysis quality initiative (ADQI) group. Crit Care. 2004;8(4):R204-12.

11. Praught ML, Shlipak MG. Are small changes in serum creatinine an important risk factor? Curr Opin Nephrol Hypertens. 2005;14(3):265-70.

12. Hoste EA, et al. RIFLE criteria for acute kidney injury are associated with hospital mortality in critically ill patients: a cohort analysis. Crit Care. 2006;10(3):R73.

13. Lewington AJ, Cerda J, Mehta RL. Raising awareness of acute kidney injury: a global perspective of a silent killer. Kidney Int. 2013;84(3):457-67.

14. Chertow GM, et al. Acute kidney injury, mortality, length of stay, and costs in hospitalized patients. J Am Soc Nephrol. 2005;16(11):3365-70.

15. Tan J, et al. Warfarin use and stroke, bleeding and mortality risk in patients with end stage renal disease and atrial fibrillation: a systematic review and meta-analysis. BMC Nephrol. 2016;17(1):157.

16. Moher D, et al. Preferred reporting items for systematic reviews and metaanalyses: the PRISMA statement. PLoS Med. 2009;6(7):e1000097.

17. Susantitaphong P, et al. World incidence of AKl: a meta-analysis. Clin J Am Soc Nephrol. 2013:8(9):1482-93.

18. Wells GA, S.B., O'Connell D, Peterson J, Welch V, Losos M, et al. The Newcastle-Ottawa Scale (NOS) for assessing the quality if nonrandomized studies in meta-analyses. [cited 2017 03/03/2017]; Available from: http:// www.ohri.ca/programs/clinical epidemiology/oxford.asp.

19. Covic A, et al. The impact of acute kidney injury on short-term survival in an eastern European population with stroke. Nephrol Dial Transplant. 2008; 23(7):2228-34

20. Khatri $M$, et al. Acute kidney injury is associated with increased hospital mortality after stroke. J Stroke Cerebrovasc Dis. 2014;23(1):25-30.

21. Lin S, et al. Characteristics, treatment and outcome of ischemic stroke with atrial fibrillation in a Chinese hospital-based stroke study. Cerebrovasc Dis. 2011;31(5):419-26.

22. Mohamed W, et al. Which comorbidities and complications predict ischemic stroke recovery and length of stay? Neurologist. 2015;20(2):27-32.

23. Saeed $F$, et al. Acute renal failure is associated with higher death and disability in patients with acute ischemic stroke: analysis of nationwide inpatient sample. Stroke. 2014:45(5):1478-80.

24. Saeed F, et al. Acute renal failure worsens in-hospital outcomes in patients with intracerebral hemorrhage. J Stroke Cerebrovasc Dis. 2015;24(4):789-94.

25. Tsagalis $\mathrm{G}$, et al. Long-term prognosis of acute kidney injury after first acute stroke. Clin J Am Soc Nephrol. 2009;4(3):616-22.

26. Nadkarni GN, et al. Dialysis requiring acute kidney injury in acute cerebrovascular accident hospitalizations. Stroke. 2015;46(11):3226-31.

27. Aho K, et al. Cerebrovascular disease in the community: results of a $\mathrm{WHO}$ collaborative study. Bull World Health Organ. 1980;58(1):113-30.

28. Adams HP Jr, et al. Classification of subtype of acute ischemic stroke. Definitions for use in a multicenter clinical trial. TOAST. Trial of org 10172 in acute stroke treatment. Stroke. 1993:24(1):35-41.

29. Waikar SS, et al. Validity of international classification of diseases, ninth revision, clinical modification codes for acute renal failure. J Am Soc Nephrol. 2006;17(6):1688-94

30. Jannot AS, et al. The diagnosis-wide landscape of hospital-acquired AKI. Clin J Am Soc Nephrol. 2017;12(6):874-84

31. Silver SA, Chertow GM. The economic consequences of acute kidney injury. Nephron. 2017.

32. Bedford $\mathrm{M}$, et al. What is the real impact of acute kidney injury? BMC Nephrol. 2014;15:95 
33. Lassnigg A, et al. Minimal changes of serum creatinine predict prognosis in patients after cardiothoracic surgery: a prospective cohort study. J Am Soc Nephrol. 2004;15(6):1597-605.

34. Lozano R, et al. Global and regional mortality from 235 causes of death for 20 age groups in 1990 and 2010: a systematic analysis for the global burden of disease study 2010. Lancet. 2012;380(9859):2095-128.

35. Silverwood RJ, et al. Cognitive and kidney function: results from a British birth cohort reaching retirement age. PLoS One. 2014;9(1):e86743.

36. Coresh J, et al. Prevalence of chronic kidney disease in the United States. JAMA. 2007;298(17):2038-47.

37. Levy EM, Viscoli CM, Horwitz RI. The effect of acute renal failure on mortality. A cohort analysis. JAMA. 1996;275(19):1489-94.

38. Wang $\mathrm{HE}$, et al. Acute kidney injury and mortality in hospitalized patients. Am J Nephrol. 2012;35(4):349-55.

39. Coca SG, et al. Long-term risk of mortality and other adverse outcomes after acute kidney injury: a systematic review and meta-analysis. Am J Kidney Dis. 2009;53(6):961-73.

40. Karkouti K, et al. Acute kidney injury after cardiac surgery: focus on modifiable risk factors. Circulation. 2009;119(4):495-502.

41. Bihorac $A$, et al. Long-term risk of mortality and acute kidney injury during hospitalization after major surgery. Ann Surg. 2009;249(5):851-8.

Ready to submit your research? Choose BMC and benefit from:

- fast, convenient online submission

- thorough peer review by experienced researchers in your field

- rapid publication on acceptance

- support for research data, including large and complex data types

- gold Open Access which fosters wider collaboration and increased citations

- maximum visibility for your research: over $100 \mathrm{M}$ website views per year

At BMC, research is always in progress.

Learn more biomedcentral.com/submissions 